\section{Summary}

Kidney brush border membrane vesicles (BBMV) are extensively used as a tool for the identification and characterization of transport systems present in the apical membrane of proximal tubular cells. Recently, differences in the transport properties of BBMV prepared by the two most popular techniques which involve either a calcium or magnesium precipitation step have been reported $[1,2]$. The present experiments were designed to examine some of the parameters responsible for the different transport properties of these preparations, namely: (1) their membrane physical state, estimated by fluorescence polarization of 1,6 -diphenyl-1,3,5-hexatriene; (2) their lipid composition; (3) their permeability to non-electrolytes.

Using rat kidney preparations of similar degree of enrichment in marker enzymes, we demonstrate that the viscosity of BBMV prepared with $\mathrm{Ca}^{2+}$ is significantly higher than that of BBMV prepared via $\mathrm{Mg}^{2+}$ precipitation (figure $I$ ). The viscosity of both preparations is unaffected by the addition of EDTA ( $10 \mathrm{mM})$ and their lipid composition, in particular their lysophosphatidylcholine content, is similar (table I). Considering the viscosity properties, permeability experiments led to an unexpected result: the passive permeability to non-electrolytes of BBMV prepared using $\mathrm{Ca}^{2+}$ is significantly higher than that of BBMV obtained through

\title{
Calcium, magnésium et bordure en brosse rénale
}

\author{
Daniel Lajeunesse, Marie-Cécile Giocondi, \\ Serge Carrière, Christian Le Grimellec
}

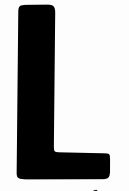

es fragments de membranes cellulaires formant de microscopiques vésicules sont couramment utilisés pour étudier le transport membranaire de différentes molécules. L'utilisation de vésicules d'origine rénale ou intestinale a permis de réaliser, au cours des dernières années, des progrès considérables dans la compréhension des mécanismes moléculaires du transport des ions, des sucres et des acides aminés. Comme pour l'intestin, la plupart des méthodes de purification des vésicules de bordure en brosse du cortex rénal reposent sur la précipitation par des cations divalents des membranes contaminantes. Selon les laboratoires, le calcium ou le magnésium sont actuellement indifféremment utilisés lors de cette étape de précipitation $[1,3]$. Les travaux réalisés sur les membranes artificielles ainsi que sur diverses membranes biologiques ont démontré que ces deux cations pouvaient affecter de façon différente certaines propriétés membranaires comme la fusion, les phénomènes de séparation de phases lipidiques, ou l'activation des phospholipases
$[4,5]$. Ces observations suggèrent que les propriétés physico-chimiques, transport inclus, des bordures en brosse rénales obtenues à l'aide de l'un ou l'autre de ces cations puissent ne pas être identiques. De fait, Biber et al.[I] ont mentionné que le transport de glucose dépendant du sodium était mieux préservé dans les vésicules obtenues par précipitation au magnésium. Récemment, Sabolic et Burckhardt [2] ont également décrit l'existence de différences marquées dans la conductance aux ions $\mathrm{H}^{+}$et $\mathrm{K}^{+}$ainsi que dans les caractéristiques du système d'échange $\mathrm{Na}^{+}-\mathrm{H}^{+}$selon le type de préparation $\left(\mathrm{Ca}^{2+}\right.$ ou $\left.\mathrm{Mg}^{2+}\right)$. Ainsi, certaines des propriétés des systèmes de transport étudiés sur les vésicules seraient liées à la méthode de préparation utilisée. Dans cette note, nous rapportons les résultats d'une étude destinée à rechercher, au niveau lipidique, les bases moléculaires de telles différences dans les propriétés de transport. A cette fin, nous avons comparé les propriétés physicochimiques des bordures en brosse rénales préparées par précipitation au chlorure de calcium ou au chlo- 
$\mathrm{Mg}^{2+}$ precipitation (figure 2). This result can account, at least partly, for the differences in transport properties previously reported $[1,2]$. To reconcile viscosity, lipid composition and permeability data, we propose that $\mathrm{Ca}^{2+}$ induces modifications in lipid-lipid/lipid-protein interactions (phase separations) or phase $\mathrm{H}_{\mathrm{II}}$ formation thus resulting in molecular defect in the BBMV membrane structure.

\section{ADRESSE}

D. Lajcunesse, S. Carric̀re : Groupe de transport membranairc, Université de Montréal, Montréal, QUE, Canada $\mathrm{H}_{3} \mathrm{C}_{3} \mathrm{~J} 7$.

M. C. Giocondi, Ch. Le Grimellec : Inserm U 251, faculté Xavier-Bichat, i6, rue HenriHuchard, 75018 Paris.

\section{TIRÉS A PART}

Ch. Le Grimellec: Inserm U 251, faculté Xavier-Bichat, 16, rue Henri-Huchard, 75018 Paris.

\section{Remerciements}

Ce traviail a bénéficié en partie du soutien financier du Conseil de Recherches Médicales du Canada er de la Fondation Canadienne des Maladies du Rein. Nous remercions Franfoise Carlier pour son aide dans la préparation du manuscrit.

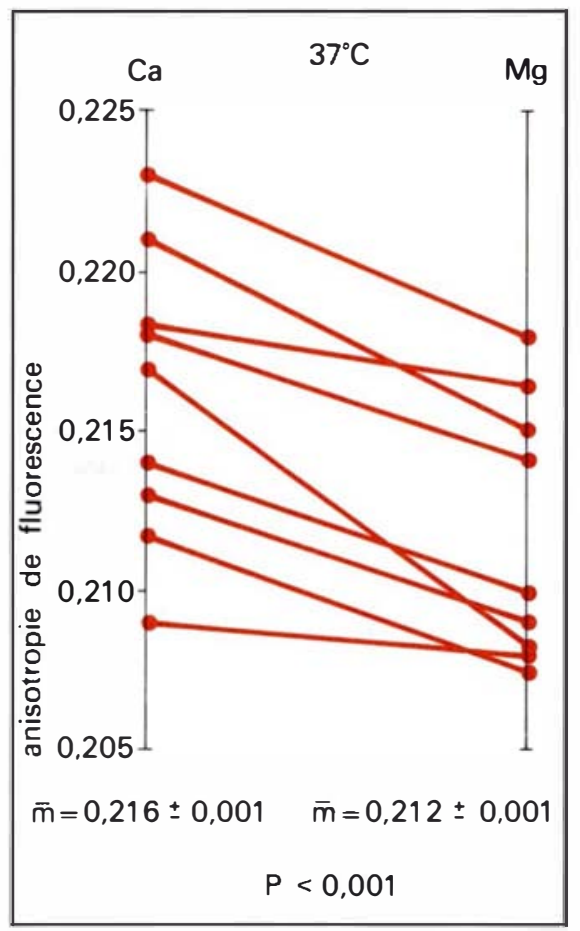

$m / s n^{0}>$ novembre 85 rure de magnésium. I.a comparaison porte sur trois aspects : l'état physique de la membrane, défini par sa fluidité et déterminé par dépolarisation de fluorescence du 1,6-diphényl-1,3,5-hexatriene (DPH) [6], sa composition en lipides et ses propriétés de perméabilité passive aux non électrolytes.

Les résultats illustrés par la figure I démontrent que la fluidité, inversement proportionnelle à l'anisotropie de fluorescence [7] des bordures en brosse rénales préparées par précipitation par le $\mathrm{CaCl}_{2}$, est significativement plus faible que celle des membranes obtenues via le $\mathrm{MgCl}_{2}$. Des résultats comparables sont obtenus pour les mesures effectuées à $25^{\circ} \mathrm{C}$, l'anisotropie moyenne des membranes préparées par le $\mathrm{CaCl}_{2}$ $(m=0,263 \pm 0,002)$ étant significativement plus élevée que celle des membranes préparées par le $\mathrm{MgCl}_{2}$ $(m=0,259 \pm 0,002, \quad p<0,01, \quad n=9$ échantillons regroupés par paires). L'addition d'EDTA (10 mM) ne modifie pas significativement les valeurs obtenues pour chaque type de préparation. Traduites en termes de viscosité relative, ces différences d'anisotropie indiquent qu'à $37^{\circ} \mathrm{C}$, comme à $25^{\circ} \mathrm{C}$, la viscosité des membranes préparées par le $\mathrm{Ca}^{2+}$ est de 4 à $5 \%$ supérieure à celle des membranes préparées par le $\mathbf{M g}^{2+}$. De telles variations sont du même ordre de grandeur que celles observées, par exemple, sous l'effet de diverses hormones [8-11]. L'addition de $10 \mathrm{mM}$ de $\mathrm{CaCl}_{2}$ à des vésicules préparées par précipitation au magnésium entraîne une augmentation immédiate de l'anisotropie de fluorescence qui passe alors de $0,211 \pm 0,002$ à $0,219 \pm 0,002\left(37^{\circ} \mathrm{C}\right.$ trois préparations différentes). La valeur ainsi obtenue reste stable dans le temps.

Une des raisons possibles de la différence d'état physique observée serait une modification de la composition en lipides des membranes, liée plus particulièrement à l'activation d'une phospholipase de type A par les ions calcium. Une telle possibilité semble toutefois exclue par l'examen de la composition en lipides des deux types de préparation : les contenus en cholestérol et la distribution des différentes classes de phospholipides (notamment la lysophosphatidylcholine) des membranes préparées par précipitation

Figure 1. Influence de l'ion précipitant sur la viscosité membranaire. Les bordures en brosse sont préparées à partir d'une suspension tubulaire $[2 I]$ issue du cortex rénal de 3 à 5 rats Sprague-Dawley pesant 250-350g. La technique de précipitation au calcium utilisée est semblable à celle décrite par Schmitz et al. [3]. Pour la préparation utilisant le magnésium, outre le remplacement $d u \mathrm{Ca}^{2+}$ par le $\mathrm{Mg}^{2+}$, on effectue simplement deux précipitations successives par le $\mathrm{MgCl}_{2}($ I $2 \mathrm{mM})$, au lieu d'une seule dans la technique $C a^{2+}$. La pureté des deux types de préparation (9 préparations), testée par l'enrichissement relatif des marqueurs enzymatiques $/ 6 /$, est comparable: Phosphatase alcaline $16,2 \pm 2,4\left(\mathrm{Ca}^{2+}\right)$ contre $16,7 \pm 1,4\left(\mathrm{Mg}^{2+}\right)$; Succinate déshydrogénase $<0,1\left(\mathrm{Ca}^{2+}\right.$ et $\left.\mathrm{Mg}^{2+}\right) ;$ Glucose 6 phosphatase $0,9 \pm 0,3$ $\left(\mathrm{Ca}^{2+}\right)$ contre $\mathrm{I,0} \pm 0,3\left(\mathrm{Mg}^{2+}\right) ;\left(\mathrm{Na}^{+}+K^{+}\right)$ATPase $\mathrm{I}, \mathrm{I} \pm 0,5\left(\mathrm{Ca}^{2+}\right)$ contre

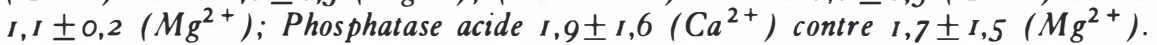
Les membranes ainsi obtenues sont marquées par le diphénylhexatriène selon les procédés déjà décrits $/ 6 \mathrm{~J}$. Les résultats sont exprimés sous la forme d'anisotropie de fluorescence ( $\mathrm{r}$ ) avec : $\mathrm{r}=\left[I_{\| /}-I_{\perp}\right] /\left[I_{\| \mid}+2 I_{\perp}\right]$. $I_{||}$et $I_{\perp}$ représentent respectivement l'intensité de la fluorescence mesurée dans un plan parallèle et perpendiculaire au plan de polarisation de la lumière excitatrice. $\mathrm{r}$ est proportionnel à la viscosité de la membrane, des valeurs plus élevées indiquant une viscosité plus importante. Chaque point représente la moyenne de 4 mesures réalisées sur une préparation membranaire provenant de 3 à 5 animaux. Les valeurs moyennes sont données $\pm S E M$. 
au $\mathrm{Ca}^{2+}$, ne sont pas significativement différents de ceux obtenus pour les membranes purifiées à l'aide du $\mathrm{Mg}^{2+}$ (tableau I). Ces compositions sont très proches de celles décrites récemment par Hise et al. pour des animaux de souche identique [12]. L'absence de stimulation marquée de phospholipases $\mathrm{A}$ par le $\mathrm{Ca}^{2+}$ dans les bordures en brosse rénales constraste avec les résultats décrits pour les bordures en brosse intestinales [13]. Elle s'accorde toutefois avec l'observation selon laquelle, pour les bordures en brosse rénales, l'addition de mépacrine, un inhibiteur de la phospholipase $A_{2}$, lors de la préparation par le $\mathrm{CaCl}_{2}$, n'atténue pas les différences avec les préparations par le $\mathrm{MgCl}_{2}$ en ce qui concerne l'échange $\mathrm{Na}^{+}-\mathrm{H}^{+}$ou encore la conductance ionique [2].

La perméabilité des membranes épithéliales à la plupart des nonélectrolytes est, comme pour les autres membranes biologiques, proportionnelle à leur coefficient de partage huile/eau [14]. Cette perméabilité est, en particulier, modulée par la composition et la configuration des lipides membranaires [15]. Les résultats illustrés par la figure 2 indiquent que la perméabilité au L-glucose ou à l'érythritol des bordures en brosse préparées avec le $\mathrm{Ca}^{2+}$ est significativement supérieure $(p<0,01)$ à celle des membranes obtenues par le $\mathrm{Mg}^{2+}$. Des expériences similaires avec ${ }^{3} \mathrm{H}$-mannitol conduisent à une conclusion identique. L'addition de calcium à des membranes préparées par précipitation au $\mathrm{MgCl}_{2}$ entraîne une augmentation significative d'environ $10 \%$ de leur perméabilité passive à l'érythritol $\left(\times_{I, I} 8\right.$; $n=43 ; \quad p<0,001), \quad$ au L-glucose ( $\times 1,09 ; n=34 ; \quad p<0,01)$ et au mannitol ( $\left.\times_{I}, 08 ; n=26 ; p<0,01\right)$. L'augmentation de perméabilité passive des vésicules purifiées par le chlorure de calcium s'accorde avec les travaux de Biber et al. [I] et de Sabolic et Burckhardt [2]. Elle permet d'expliquer en grande partie l'accumulation transitoire de glucose et les capacités d'échange électroneutre $\mathrm{Na}^{+}-\mathrm{H}^{+}$plus faibles des membranes préparées par cette méthode. Cet effet sur la perméabilité passive semble toutefois en contradiction avec les résultats de viscosité membranaire : l'on s'attendrait, en effet, à ce que les membranes $\mathrm{Ca}^{2+}$, plus rigides, aient une perméabilité passive plus faible. Cette apparente contradiction peut cependant être levée, considérant que le calcium pourrait induire dans les bordures en brosse rénales des phénomènes de séparation ou d'inversion de phase d'une fraction des phospholipides négatifs $[4,16]$, comme dans le cas de membranes

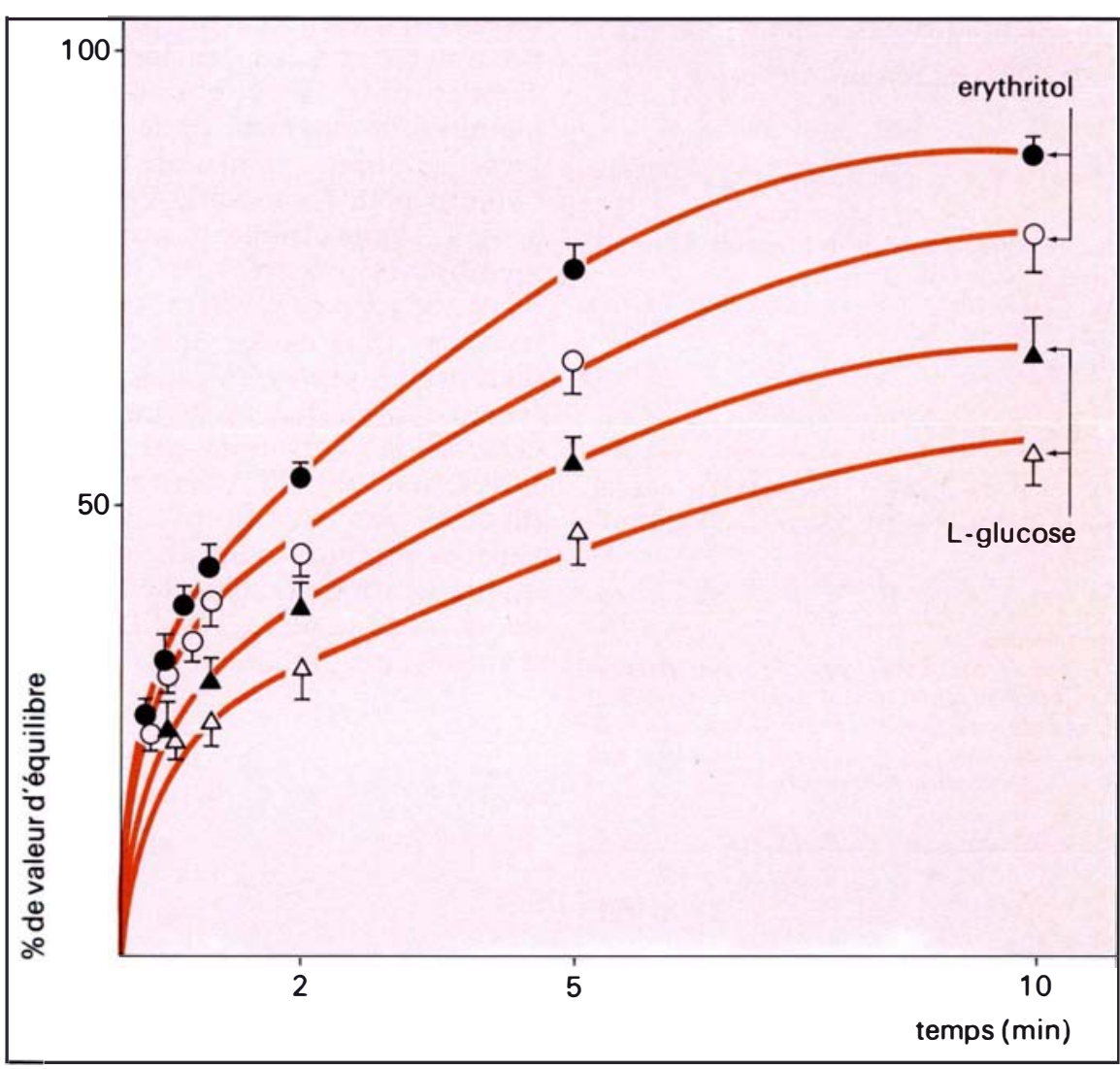

Figure 2. Perméabilité aux non-électrolytes des membranes préparées par le $\mathrm{Ca}^{2+}$ ou le $\mathrm{Mg}^{2+}$. La préparation de bordures en brosse partiellement purifiée est resuspendue dans un tampon contenant : Mannitol $100 \mathrm{mM}, \mathrm{NaCl} 100 \mathrm{mM}$, Hepes Iо $\mathrm{mM}, \mathrm{pH} 7,40$. Les bordures en brosse purifiées sont resuspendues (IO-20 mg protéines/ml) dans ce même tampon à la fin de la préparation. Les mesures de flux débutent avec l'addition de $100 \mu \mathrm{l}$ de la solution de bordures en brosses à $500 \mu \mathrm{l}$ du milieu d'incubation. Ce milieu est de composition identique au tampon de resuspension mais contient en plus, soit $\left.0,5 \mathrm{mM} /{ }^{14} \mathrm{C}\right]-$ Erythritol, soit $\left.0,5 \mathrm{mM} /{ }^{14} \mathrm{C}\right] \mathrm{L}$-glucose. $A$ intervalles de temps fixes, $50 \mu \mathrm{l}$ de la solution sont prélevés et filtrés sur un filtre millipore $0,45 \mu \mathrm{M}$, préalablement recouvert de I $\mathrm{ml}$ de solution d'arrêt à $0^{\circ} \mathrm{C}$. Les filtres sont rincés par $6 \mathrm{ml}$ de solution d'arrêt, séchés et placés dans des fioles à scintillation pour le comptage de la radioactivité. Les solutions d'arrêt sont constituées du tampon de resuspension auquel to $\mathrm{mM}$ d'érythritol ou de L-glucose ont été ajoutés. Les résultats érythritol portent sur 8 préparations de membranes différentes, traitées en parallele par le $\mathrm{Ca}^{2+}$ ou le $\mathrm{Mg}^{2+}$. Dans chaque condition expérimentale les expériences ont été réalisées en duplicate. Ceux de I-glucose proviennent de 4 préparations différentes. Les points indiquent les valeurs moyennes $\pm S E M$. O. $\triangle$ : préparations $\mathrm{Ca}^{2+} ; \mathrm{O}, \triangle:$ préparations $\mathrm{Mg}^{2+}$. 


\begin{tabular}{|c|c|c|}
\hline \multicolumn{3}{|c|}{$\begin{array}{c}\text { COMPOSITION EN LIPIDES DES BORDURES EN BROSSE } \\
\text { SELON LE TYPE DE PREPARATION }\end{array}$} \\
\hline & $\mathrm{BBMV}-\mathrm{Ca}^{2+}$ & BBMV-Mg ${ }^{2+}$ \\
\hline $\begin{array}{l}\text { (mole/mole) } \\
\text { cholestérol/phospholipides }\end{array}$ & 0,57 & 0,57 \\
\hline $\begin{array}{l}\text { (mole/100mole) } \\
\text { sphingomyéline } \\
\text { phosphatidylcholine } \\
\text { phosphatidyléthanolamine } \\
\text { phosphatidylsérine+ } \\
\text { phosphatidylinositol f } \\
\text { lysophosphatidylcholine }\end{array}$ & $\begin{array}{l}29 \\
24 \\
22 \\
17 \\
1.6\end{array}$ & $\begin{array}{l}31 \\
24 \\
20 \\
20 \\
2,1\end{array}$ \\
\hline
\end{tabular}

Les bordures en brosse purifiées ont été extraites par la méthode de Bligh et Dyer $\mid 22 /$. Les contenus en cholestérol, phospholipides totaux et les différentes classes de phospholipides ont été déterminés selon des méthodes décrites précédemment $|23,24|$. Les résultats représentent les valeurs moyennes obtenues à partir de trois préparations différentes traitées en double.

d'organisation moléculaire dans la bicouche et en augmente sensiblement la perméabilité passive [17]. La formation de phases hexagonales inversées au sein de la membrane également sensible au calcium pour les mélanges de lipides contenant, outre des phospholipides négatifs, de la phosphatidyléthanolamine et du cholestérol, conduirait à un résultat équivalent. L'hypothèse d'un effet du calcium, via des modifications de microdomaines dans les bordures en brosse rénales est en accord avec les observations récentes sur les membranes d'hépatocyte [18-20] qui suggèrent fortement une hétérogénéité dans l'effet membranaire du $\mathrm{Ca}^{2+}$. Cette hypothèse n'exclut cependant pas une participation éventuelle des éléments du cytosquelette qui restent associés à la membrane lors des préparations de bordures en brosse. En conclusion, nos résultats démontrent que la rigidité et la perméabilité passive aux non-électrolytes des bordures en brosse rénales préparées à l'aide du $\mathrm{Ca}^{2+}$ excèdent celles des membranes obtenues par précipitation au $\mathrm{Mg}^{2+}$. Ils permettent d'expliquer, au moins en partie, les différences de propriété de transport rapportées dans la littérature pour les deux types de préparations. Des modifications locales dans l'organisation des phospholipides membranaires pourraient être à l'origine de ces différences
I. Biber J, Sticger B, Haase W, Murer H. A high yield preparation for rat kidney brush border membrancs. Biochim Biophys Acta 1981; 647 : I69-76.

2. Sabolic I, Burckhardt G. Effect of the preparation method on $\mathrm{Na}^{+}-\mathrm{H}^{+}$exchange an ion permeabilitics in rat renal brush-border membranes. Biochim Biophys Acta 1984; 772 : 140-8.

3. Schmitz J, Preiser H, Maestracci D, Ghosh BK, Cerda JJ, Crane RK. Purification of the human intestinal brush border membrane. Biochim Biophys Acta 1973; 323: 98-11 2.

4. Papahadjopoulos D, Portis A, Pangborn W Calcium-induced lipid phase transitions and membranc fusion. Ann NY Acad Sci 1978; 308 : 50-66.

5. Thompson GA Jr. Phospholipid metabolism in animal tissucs. In : Ansell GB, Hawthorne JN, Dawson RMC, eds. Form and Function of phospholipids, BBA Library rol 3. Amsterdam: Elsevier, I973: 67-96.

6. Le Grimellec C, Giocondi MC, Carrière B, Carrière $S$, Cardinal J. Membrane fluidity and enzyme activitics in brush border and basolateral membranes of the dog kidney. Am 3 Physiol 1982; 242: F 246-53.

7. Shinitzky M, Barenholtz Y. Fluidity parameters of lipid regions determined by fluorescence polarization. Biochim Biophys Acta 1978; 515: 367-94.

8. Master BR, Yguerabide J, Fanestil DD Microviscosity of mucosal cellular membranes in toad urinary bladder: Relation to antidiuretic hormone action on water permeability. $7 \mathrm{Membr}$ Biol 1978; 40 : 179-90.

9. Hershkowitz M, Zwiers H, Gispen WH. The effect of ACTH on rat brain synaptic plasma membrane lipid fluidity. Biochim Biophys Acta 1982; 692: 495-7.

\section{REFERENCES}

\section{RÉFÉRENCES}

Io. Goodsaid-Zaldvondo F, Rintoul DA, Carlson JC, Hansel W. I.utcolysis-induced changes in phase composition and fluidity of bovine luteal cell membranes. Proc Natl Acad Sci USA r 982; $79: 4332-6$

II. Hyslop PA, York DA, Saucrbheber RD. Effects of insulin on the lipid structure of liver plasma membrane measured with fluorescence and ESR spectroscopic methods. Biochim Biophys Acta $1984 ; 776: 267-78$.

12. Hise MK, Mantulin WW, Weinman EJ Fluidity and composition of brush border and basolateral membranes from rat kidney. $A m$ J Physiol I 984; 247 : F434-9.

13. Hauser H, Howell K, Dawson RMC, Bowyer DE. Rabbit small intestinal brush border membrane. Preparation and lipid composition. Biochim Biophys Acta 1980; 602: 567-77.

I 4. Wright EM, Pictras RJ. Routes of nonelectrolyte permeation across epithelial membranes. J. Membr Biol I 974; 17 : 293-31 2

I 5. Van Zoclen EJJ, Dc Jesus CH, De Jonge E, Mulder M, Block MC, Degier J. Non-electrolytc permeability as a tool for studying membranc fluidity. Biochim Biophys Acta I 978; 5 I I : 335-47

I6. Tilcock CPS, Bally MB, Farren SB, Cullis PR, Gruner SM. Cation-dependent segregation phenomena and phase bchaviour in model membranc systems containing phosphatidylserine: influcnce of cholesterol and acyl chain composition. Biochemistry $1984 ; 23: 2696-703$

17. Jain MK. Phasc properties of bilayers. In Jain MD, Wagner RC, eds. Introduction to biological membranes. New York: Wilcy, 1980: 53-175.

I8. Storch J, Schachter D, Inoue M, Wolk off AW. I.ipid fluidity of hepatocyte plasma membrane subfractions and their differential regulation by calcium. Biochim Biophys Acta I 983; $727:$ 209-I 2

19. Schroeder F, Soler-Argilaga C. Calcium modulates fatty acid dynamics in rat liver plasma membrancs. Eur.7 Biochem 1983; 132 : 517-24.

20. Gordon LM, Whetton AD, Rawal S, Esgate JA, Houslay MD. Perturbations of liver plasma membranes induced by $\mathrm{Ca}^{2}{ }^{+}$are detected using a fatty acid spin label and adenylate cyclase as membrane probes. Biochim Biophys Acta I983; 729: 104-1 4 .

2 I. Vinay $P$, Gougoux A, I,emieux G. Isolation of a pure suspension of rat proximal tubules. $\mathrm{Am}$ J Physiol 198I; 24I: F403-II.

22. Bligh EG, Dyer WJ. A rapid method of total lipid extraction and purification. Can $\mathcal{J}$ Biochem I 959; $37: 9$ I $1-7$.

23. Le Grimellec C, Leblanc C. Effect of membrane cholestcrol on potassium transport in mycoplasma mycoides var capri ( $\left.\mathrm{PG}_{3}\right)$. Biochim Biophys Acta 1978; 514: 152-63.

24. Le Grimellec C, Cardinal J, Giocondi MC Carrière S. Control of membrane lipids in mycoplasma gallisepticum. Effect on lipid order. J Bacteriol I981; 146 : 1 55-62. 